\title{
Autoimmune progesterone dermatitis: treatment with oophorectomy
}

\author{
S. Medeiros, R. Rodrigues-Alves,* M. Costa,† A. Afonso,‡ A. Rodrigues and J. Cardoso \\ Department of Dermatology, Curry Cabral Hospital, Lisbon; *Immunoalergology Department, Santa Maria Hôpital, Lisbon; +Obstetrics and Gynecology \\ Department, Maternidade Alfredo da Costa, Lisbon; and ¥Pathology Department, Curry Cabral Hospital, Lisbon, Portugal
}

doi:10.1111/j.1365-2230.2009.03217.x

Autoimmune progesterone dermatitis (AIPD) is a rare presentation of progesterone hypersensitivity, characterized by recurrent cyclical eruptions during the luteal phase of the menstrual cycle with a variable clinical presentation.

A 42-year-old woman was evaluated in 2001 with a dermatosis that had begun during her pregnancy the previous year, at 32 weeks of gestation. Lesions were localized to the face, neckline area, trunk and limbs, and consisted of multiple pruritic and erythematous papules and plaques with crusted erosions (Fig. 1a). The dermatitis appeared every month, 5-6 days before the onset of menses and gradually resolved 7-10 days later. The patient had given birth six times and her only medication was oral contraceptive pills (desogestrel $0.15 \mathrm{mg}$ and ethinyl oestradiol $0.02 \mathrm{mg}$, Mercilon ${ }^{\circledR}$; Organon, Lisbon, Portugal), which she had taken intermittently for 20 years.

Histopathological examination of a biopsy taken from a lesion revealed epidermal hyperplasia and perivascular inflammatory infiltration with mononuclear cells.

Three months later, she was admitted to our department because of widespread dermatitis, predominantly on the limbs (also affecting the palms and soles) and trunk, and consisting of multiple annular erythematous papules and patches with central vesiculation. On the labial mucosa there were scattered painful eroded lesions (Fig. 1b).

Histological examination of a second biopsy showed a total epidermal necrosis and perivascular superficial mononuclear infiltrate compatible with erythema multiforme diagnosis. Direct immunofluorescence was negative.

The patient was treated with prednisolone (1 mg/ $\mathrm{kg} /$ day) and antihistamines with no improvement.

Correspondence: Dr Sandra Medeiros, Dermatology Department, Curry Cabral Hospital. Rua da Beneficiência n`8, 1069-166 Lisbon, Portugal

E-mail: sandradmedeiros@clix.pt

Conflict of interest: none declared.

Accepted for publication 17 September 2008
Subsequently, she discontinued all medical care. Between November 2002 and May 2005 she reported complete clinical remission. In August 2005 she returned to our clinic because the dermatosis had reappeared and was much more severe. An intradermal test with medroxyprogesterone $50 \mathrm{mg} / \mathrm{mL}$, performed on the seventh day of the menstrual cycle, was positive 30 min after injection and persisted for $24 \mathrm{~h}$. An intradermal test with oestradiol was negative.

The patient was given treament with an intramuscular gonadotrophin-releasing hormone (GnRH) agonist (leuprorelin acetate) for 6 months, achieving amenorrhea and complete cutaneous clearing from the first month. For ethical reasons, no attempt was made to induce a flare with an intramuscular hormonal challenge. Hysterectomy and bilateral oophorectomy was performed in March 2006, followed by oestrogen supplementation. Two years after surgery, the patient remains free of symptoms.

Approximately 50 cases of AIPD have been published in the literature. The onset during pregnancy, as in our patient, is very rare and may be related to the increase in serum progesterone. Less than five cases have been reported with or without associated spontaneous abortion. ${ }^{1,2}$

The clinical features of AIPD include urticaria, angiooedema, erythema multiforme, erythema annulare centrifugum, fixed drug eruptions, stomatitis or folliculitis, and there are no specific histological features. ${ }^{3-5}$ AIPD needs to be distinguished from many other chronic dermatoses that worsen premenstrually. The diagnosis requires, in addition to recurrent cutaneous eruptions, two of the four main criteria: (i) a positive intradermal skin test with progesterone; (ii) prevention of the premenstrual flare by inhibiting ovulation; (iii) demonstration of circulating antibodies to progesterone; and (iv) reproduction of symptoms with an intramuscular hormonal challenge. ${ }^{1,5}$

It has been reported that AIPD may clear during times of amenorrhea, or evolve towards remission with treatment. Nevertheless, it is still not clear to us why 

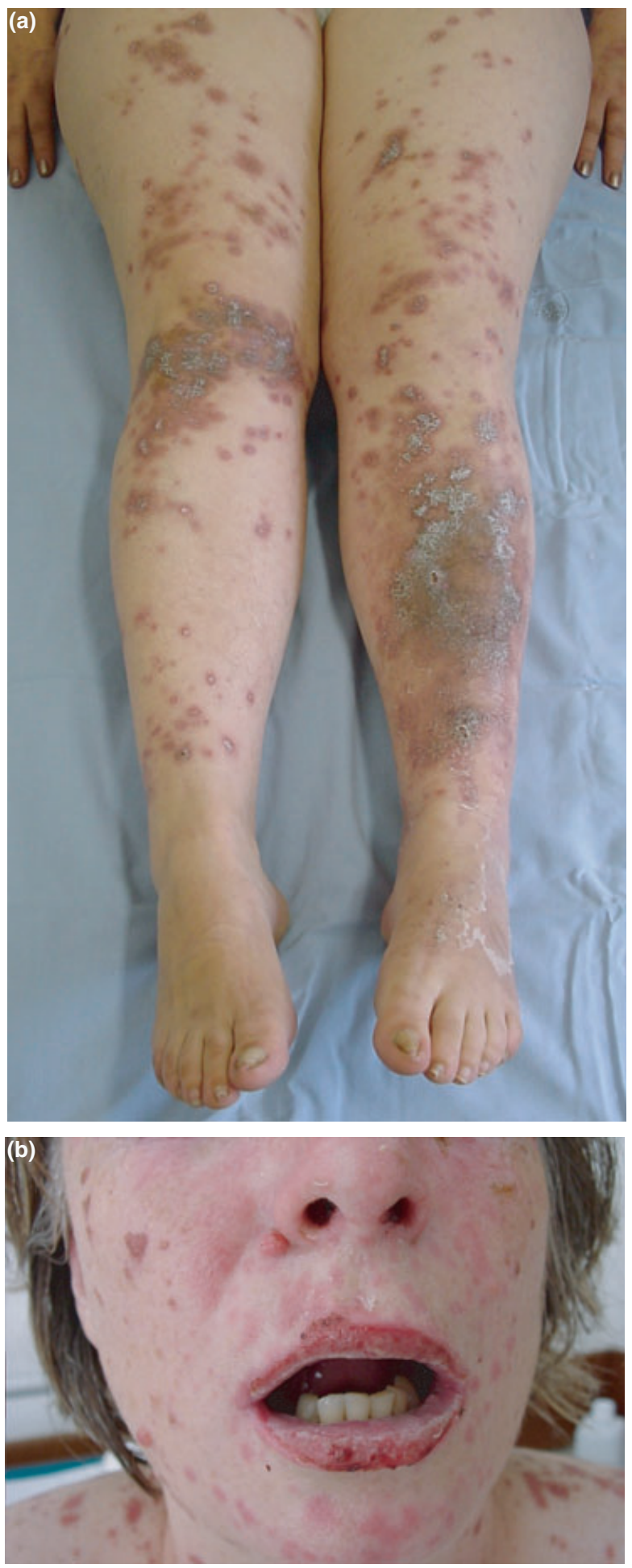

Figure 1 (a) Multiple papules and plaques with crusted erosions; (b) exudative erythema multiforme-like eruption. there was complete remission for 30 months without any treatment. Such an extended remission period has not been described in other AIPD cases.

The mainstay of treatment for AIPD is to inhibit endogenous progesterone secretion by suppressing ovulation. In our case, the GnRH analogue showed efficacy, but because of the side-effects, the clinical severity of the disease, and because the patient did not want any more children, a bilateral oophorectomy was performed. This treatment has been reported in $<15$ cases. ${ }^{3,5,6}$ Patients who have undergone this procedure have had complete resolution of their outbreaks after a series of medical options, such as oestrogens, tamoxifen, GnRH, luteinizing hormone-releasing hormone, azathioprine, prednisolone, dapsone or thalidomide have been tried. Slater et al. ${ }^{6}$ described one exception to this 'rule'; in patient 2 of their study, anaphylactoid symptoms improved after bilateral salpingoophorectomy, but the patient still required antihistamines to control flushing and bloating. In our opinion, oophorectomy should be considered a valuable option in severe cases of AIPD with difficult management, such as ours.

\section{References}

1 Stephens C. Perimenstrual eruptions. Clin Dermatol 1997; 15: $31-4$.

2 Dedecker F, Graesslin O. Autoimmune progesterone dermatitis: a rare pathology. Eur J Obstet Gynecol Reprod Biol 2005; 123: 120-1.

3 Snyder JL, Krishnaswamy G. Autoimmune progesterone dermatitis and its manifestation as anaphylaxis: a case report and literature review. Ann Allergy Asthma Immunol 2003; 90 (Suppl. 5): 469-77.

4 Cocuroccia B, Gisondi P, Gubinelli E et al. Autoimmune progesterone dermatitis. Gynecol Endocrinol 2006; 22: 54-6.

5 Vasconcelos C, Xavier P, Vieira A et al. Autoimmune progesterone urticaria. Gynecol Endocrinol 2000; 14: 245-7.

6 Slater JE, Raphael G, Cutler GB Jr et al. Recurrent anaphylaxis in menstruating women: treatment with a luteinizing hormone-releasing hormone agonist - a preliminary report. Obstet Gynecol 1987; 70 (Suppl. 4): 542-6. 